\title{
Linearization of Second-Order Ordinary Differential Equations by Generalized Sundman Transformations
}

Warisa NAKPIM and Sergey V. MELESHKO

School of Mathematics, Institute of Science, Suranaree University of Technology, Nakhon Ratchasima, 30000, Thailand

E-mail:warisa_2006@hotmail.com,sergey@math.sut.ac.th

Received January 18, 2010, in final form June 03, 2010; Published online June 15, 2010 doi:10.3842/SIGMA.2010.051

\begin{abstract}
The linearization problem of a second-order ordinary differential equation by the generalized Sundman transformation was considered earlier by Duarte, Moreira and Santos using the Laguerre form. The results obtained in the present paper demonstrate that their solution of the linearization problem for a second-order ordinary differential equation via the generalized Sundman transformation is not complete. We also give examples which show that the Laguerre form is not sufficient for the linearization problem via the generalized Sundman transformation.
\end{abstract}

Key words: linearization problem; generalized Sundman transformations; nonlinear secondorder ordinary differential equations

2010 Mathematics Subject Classification: 34A05; 34A25

\section{Introduction}

The basic problem in the modeling of physical and other phenomena is to find solutions of differential equations. Many methods of solving differential equations use a change of variables that transforms a given differential equation into another equation with known properties. Since the class of linear equations is considered to be the simplest class of equations, there arises the problem of transforming a given differential equation into a linear equation. This problem is called a linearization problem ${ }^{1}$.

The linearization problem of a second-order ordinary differential equation via point transformations was solved by Sophus Lie [3]. He also noted that all second-order ordinary differential equations can be mapped into each other by means of contact transformations. Hence, the solution of the linearization problem via contact transformations is trivial.

Comparing with the set of contact transformations the set of generalized Sundman transformations is weaker: not any second-order ordinary differential equation can be transformed to a linear equation. Hence, it is interesting to study an application of the set of generalized Sundman transformations to the linearization problem of second-order ordinary differential equations.

The linearization problem via a generalized Sundman transformation for second-order ordinary differential equations was investigated in [4]. The authors of [4] obtained that any second-order linearizable ordinary differential equation which can be mapped into the equation $u^{\prime \prime}=0$ via a generalized Sundman transformation has to be of the form

$$
y^{\prime \prime}+\lambda_{2}(x, y) y^{\prime 2}+\lambda_{1}(x, y) y^{\prime}+\lambda_{0}(x, y)=0 .
$$

Using the functions

$$
\lambda_{3}=\lambda_{1 y}-2 \lambda_{2 x}, \quad \lambda_{4}=2 \lambda_{0 y y}-2 \lambda_{1 x y}+2 \lambda_{0} \lambda_{2 y}-\lambda_{1 y} \lambda_{1}+2 \lambda_{0 y} \lambda_{2}+2 \lambda_{2 x x},
$$

\footnotetext{
${ }^{1}$ The linearization problem has been studied in many publications. A short review can be found in $[1,2]$.
} 
they showed that equation (1) can be mapped into the equation $u^{\prime \prime}=0$ via a generalized Sundman transformation if the coefficients $\lambda_{i}(x, y)(i=0,1,2)$ satisfy the conditions:

(a) if $\lambda_{3}=0$, then $\lambda_{4}=0$;

(b) if $\lambda_{3} \neq 0$, then $\lambda_{4} \neq 0$ and the following equations have to be satisfied

$$
\begin{aligned}
& \lambda_{4}^{2}+2 \lambda_{3 x} \lambda_{4}-2 \lambda_{3}^{2} \lambda_{1 x}+4 \lambda_{3}^{2} \lambda_{0 y}+4 \lambda_{3}^{2} \lambda_{0} \lambda_{2}-2 \lambda_{3} \lambda_{4 x}-\lambda_{3}^{2} \lambda_{1}^{2}=0, \\
& \lambda_{3 y} \lambda_{4}+\lambda_{3}^{2} \lambda_{1 y}-2 \lambda_{3}^{2} \lambda_{2 x}-\lambda_{3} \lambda_{4 y}=0 .
\end{aligned}
$$

The generalized Sundman transformation was also applied $[5,6]$ for obtaining necessary and sufficient conditions for a third-order ordinary differential equation to be equivalent to a linear equation in the Laguerre form. Some applications of the generalized Sundman transformation to ordinary differential equations were considered in [7] and earlier papers, which are summarized in the book [8].

According to the Laguerre theorem in any linear ordinary differential equation the two terms of order below next to highest can be simultaneously removed by a point transformation. For example, the Laguerre form of a second-order ordinary differential equation is the linear equation $u^{\prime \prime}=0$. For obtaining this form, several point transformations are applied consecutively. Since the composition of point transformations is a point transformation, the final transformation is again a point transformation. This is not the case for generalized Sundman transformations: the composition of a point transformation and a generalized Sundman transformation is not necessarily a generalized Sundman transformation. Hence, for the linearization problem via generalized Sundman transformations it is not sufficient to use the Laguerre form.

In this paper, we demonstrate that the solution of the linearization problem via the generalized Sundman transformation of second-order ordinary differential equations given in [4] only gives particular criteria for linearizable equations. Complete analysis of the compatibility of arising equations is given for the case $F_{x}=0$.

\section{Generalized Sundman transformations}

A generalized Sundman transformation is a non-point transformation defined by the formulae

$$
u(t)=F(x, y), \quad d t=G(x, y) d x, \quad F_{y} G \neq 0 .
$$

Let us explain how the generalized Sundman transformation maps one function into another.

Assume that $y_{0}(x)$ is a given function. Integrating the second equation of (2), one obtains $t=Q(x)$, where

$$
Q(x)=t_{0}+\int_{x_{0}}^{x} G\left(s, y_{0}(s)\right) d s
$$

with some initial conditions $t_{0}$ and $x_{0}$. Using the inverse function theorem, one finds $x=Q^{-1}(t)$. Substituting $x$ into the function $F\left(x, y_{0}(x)\right)$, one gets the transformed function

$$
u_{0}(t)=F\left(Q^{-1}(t), y_{0}\left(Q^{-1}(t)\right)\right) .
$$

Conversely, let $u_{0}(t)$ be a given function of $t$. Using the inverse function theorem, one solves the equation

$$
u_{0}(t)=F(x, y)
$$

with respect to $y: y=\phi(x, t)$. Solving the ordinary differential equation

$$
\frac{d t}{d x}=G(x, \phi(x, t)),
$$


one finds $t=H(x)$. The function $H(x)$ can be written as an action of a functional $H=\mathcal{L}\left(u_{0}\right)$. Substituting $t=H(x)$ into the function $\phi(x, t)$, the transformed function $y_{0}(x)=\phi(x, H(x))$ is obtained.

Notice that for the case $G_{y}=0$ the action of the functional $\mathcal{L}$ does not depend on the function $u_{0}(t)$. In this case the generalized Sundman transformation becomes a point transformation. Conversely, since for a point transformation the value $d t$ in the generalized Sundman transformation is the total differential of $t$, then the compatibility condition for $d t$ to be a total differential leads to the equation $G_{y}=0$. Hence, the generalized Sundman transformation is a point transformation if and only if $G_{y}=0$.

Formulae (2) also allow us to obtain the derivatives of $u_{0}(t)$ through the derivatives of the function $y_{0}(x)$, and vice versa.

Hence, using transformation (2), one can relate the solutions of two differential equations $Q\left(x, y, y^{\prime}, \ldots, y^{(n)}\right)=0$ and $P\left(t, u, u^{\prime}, \ldots, u^{(n)}\right)=0$. Therefore the knowledge of the general solution of one of them gives the general solution of the other equation, up to solving one ordinary differential equation of first-order and finding two inverse functions.

\section{Necessary conditions}

We start with obtaining necessary conditions for the linearization problem.

First, one finds the general form of a second-order ordinary differential equation

$$
y^{\prime \prime}=H\left(x, y, y^{\prime}\right)
$$

which can be mapped via a generalized Sundman transformation into the linear equation

$$
u^{\prime \prime}+\beta u^{\prime}+\alpha u=\gamma,
$$

where $\alpha(t), \beta(t)$ and $\gamma(t)$ are some functions. Notice that the Laguerre form of a linear secondorder ordinary differential equation corresponds to $\alpha=0, \beta=0$ and $\gamma=0$.

The function $u$ and its derivatives $u^{\prime}$ and $u^{\prime \prime}$ are defined by the first formula (2) and its derivatives with respect to $x$ :

$$
\begin{aligned}
& u^{\prime} G=F_{x}+F_{y} y^{\prime}, \\
& u^{\prime \prime} G^{2}+u^{\prime}\left(G_{x}+G_{y} y^{\prime}\right)=F_{y} y^{\prime \prime}+2 F_{x y} y^{\prime}+F_{y y} y^{2}+F_{x x} .
\end{aligned}
$$

The independent variable $t$ is defined by the functional $\mathcal{L}(u)$. As noted above, if $G_{y} \neq 0$, then the action of the functional $\mathcal{L}$ depends on the function $u$. Hence, if one of the coefficients (3) is not constant and $G_{y} \neq 0$, then the substitution of $t$ into equation (3) gives a functional equation. Since the case $G_{y}=0$ reduces the generalized Sundman transformation to a point transformation $^{2}$, the irreducible generalized Sundman transformation maps equation (3) into a differential equation only for constant coefficients $\alpha, \beta$ and $\gamma$. Thus, finding the derivatives $u^{\prime}, u^{\prime \prime}$ from (4), and substituting them into (3) with constant coefficients, one has the following equation

$$
y^{\prime \prime}+\lambda_{2}(x, y) y^{\prime 2}+\lambda_{1}(x, y) y^{\prime}+\lambda_{0}(x, y)=0,
$$

where the coefficients $\lambda_{i}(x, y)(i=0,1,2)$ are related to the functions $F$ and $G$ :

$$
\lambda_{2}=\left(F_{y y} G-F_{y} G_{y}\right) / K,
$$

\footnotetext{
${ }^{2}$ Later it will be shown that in our study for $F_{x}=0$ this case is automatically excluded from consideration because in the process of studying the compatibility this case leads to the conditions $\lambda_{3}=0$ and $\lambda_{4}=0$, which were considered in [4].
} 


$$
\begin{aligned}
& \lambda_{1}=\left(2 F_{x y} G-F_{x} G_{y}-F_{y} G_{x}+F_{y} \beta G^{2}\right) / K, \\
& \lambda_{0}=\left(F_{x x} G-F_{x} G_{x}+F_{x} \beta G^{2}+\alpha F G^{3}-G^{3} \gamma\right) / K,
\end{aligned}
$$

where $K=G F_{y} \neq 0$.

Equation (5) presents the necessary form of a second-order ordinary differential equation which can be mapped into a linear equation (3) via a generalized Sundman transformation.

\section{Sufficient conditions}

For obtaining sufficient conditions, one has to solve the compatibility problem (6)-(8), considering (6)-(8) as an overdetermined system of partial differential equations for the functions $F$ and $G$ with the given coefficients $\lambda_{i}(x, y)(i=0,1,2)$. Notice that the compatibility conditions (6)-(8) for the particular case $\alpha=0, \beta=0$ and $\gamma=0$ were obtained in [4]. This case corresponds to the Laguerre form of a linear second-order ordinary differential equation. It is shown here that for the linearization problem via generalized Sundman transformations it is not sufficient to use the Laguerre form.

The compatibility analysis depends on the value of $F_{x}$. A complete study of all cases is cumbersome. Here a complete solution is given for the case where $F_{x}=0$.

Solving equations (6)-(8) with respect to $F_{y y}, \beta$ and $\gamma$, one finds

$$
\begin{aligned}
& F_{y y}=\left(G_{y} F_{y}+F_{y} G \lambda_{2}\right) / G, \\
& \beta=\left(G_{x}+G \lambda_{1}\right) / G^{2}, \\
& \gamma=\left(-F_{y} \lambda_{0}+\alpha F G^{2}\right) / G^{2} .
\end{aligned}
$$

Since $F_{x}=0$, then differentiating $F_{y y}$ with respect to $x$, one obtains

$$
G G_{x y}-G_{x} G_{y}+\lambda_{2 x} G^{2}=0 .
$$

Differentiating (10) and (11) with respect to $x$ and $y$, one obtains the following equations

$$
\begin{aligned}
& G_{x x}=\left(2 G_{x}^{2}+G_{x} G \lambda_{1}-\lambda_{1 x} G^{2}\right) / G, \\
& G_{x y}=G \lambda_{3}-G_{y} \lambda_{1}, \\
& 2 G_{x} \lambda_{0}-\lambda_{0 x} G=0, \\
& \alpha=\left(-G_{y} \lambda_{0}+G\left(\lambda_{0 y}+\lambda_{0} \lambda_{2}\right)\right) / G^{3},
\end{aligned}
$$

where

$$
\lambda_{3}=\lambda_{1 y}-2 \lambda_{2 x} .
$$

Substituting (14) into (12), this becomes

$$
G_{x} G_{y}+G_{y} G \lambda_{1}-G^{2}\left(\lambda_{2 x}+\lambda_{3}\right)=0 .
$$

Comparing the mixed derivatives $\left(G_{x y}\right)_{x}=\left(G_{x x}\right)_{y}$, one obtains the equation

$$
G_{x} \lambda_{3}-G\left(\lambda_{2 x x}+\lambda_{2 x} \lambda_{1}+\lambda_{3 x}\right)=0 .
$$

Differentiating $\alpha$ with respect to $x$ and $y$, one has

$$
\begin{aligned}
& 2 G_{x}\left(\lambda_{0 y}+\lambda_{0} \lambda_{2}\right)+G_{y}\left(\lambda_{0 x}+2 \lambda_{0} \lambda_{1}\right)-G\left(\lambda_{0 x y}+\lambda_{0 x} \lambda_{2}+4 \lambda_{2 x} \lambda_{0}+2 \lambda_{0} \lambda_{3}\right)=0, \\
& 2 G G_{y y} \lambda_{0}-6 G_{y}^{2} \lambda_{0}+2 G_{y} G\left(3 \lambda_{0 y}+2 \lambda_{0} \lambda_{2}\right)-G^{2}\left(\lambda_{4}+2 \lambda_{5}-\lambda_{1} \lambda_{3}\right)=0
\end{aligned}
$$

where

$$
\begin{aligned}
& \lambda_{4}=2 \lambda_{0 y y}-2 \lambda_{1 x y}+2 \lambda_{0} \lambda_{2 y}-\lambda_{1 y} \lambda_{1}+2 \lambda_{0 y} \lambda_{2}+2 \lambda_{2 x x} \\
& \lambda_{5}=\lambda_{2 x x}+\lambda_{2 x} \lambda_{1}+\lambda_{3 x}+\lambda_{1} \lambda_{3} .
\end{aligned}
$$

Further analysis of the compatibility depends on $\lambda_{3}$. 


\subsection{Case $\lambda_{3} \neq 0$}

From equations (18), one finds

$$
G_{x}=G\left(\lambda_{2 x x}+\lambda_{2 x} \lambda_{1}+\lambda_{3 x}\right) / \lambda_{3} .
$$

Substituting $G_{x}$ into equations (15), (17), (13) and (14), one obtains the equations

$$
\begin{aligned}
& \lambda_{0 x}=2 \lambda_{0}\left(-\lambda_{1} \lambda_{3}+\lambda_{5}\right) / \lambda_{3}, \\
& \lambda_{2 x x y}=-\lambda_{2 x y} \lambda_{1}-\lambda_{3 x y}-2 \lambda_{2 x}^{2}-2 \lambda_{2 x} \lambda_{3}-\lambda_{3 y} \lambda_{1}+\left(\lambda_{3 y} \lambda_{5}\right) \lambda_{3}^{-1}, \\
& \lambda_{2 x x x}=-\lambda_{3 x x}-\lambda_{1 x} \lambda_{2 x}-\lambda_{1 x} \lambda_{3}+\lambda_{2 x} \lambda_{1}^{2}+\lambda_{1}^{2} \lambda_{3}-2 \lambda_{1} \lambda_{5}+\lambda_{3}^{-1} \lambda_{5}\left(\lambda_{3 x}+\lambda_{5}\right), \\
& G_{y} \lambda_{5}-G \lambda_{3}\left(\lambda_{2 x}+\lambda_{3}\right)=0 .
\end{aligned}
$$

\subsubsection{Case $\lambda_{5} \neq 0$}

Equation (25) gives

$$
G_{y}=G \lambda_{3}\left(\lambda_{2 x}+\lambda_{3}\right) / \lambda_{5}
$$

Substituting $G_{y}$ into equations (14), (19) and (20) and comparing the mixed derivatives $\left(G_{x}\right)_{y}=$ $\left(G_{y}\right)_{x}$, one gets

$$
\begin{aligned}
& \lambda_{3} \lambda_{5}\left(6 \lambda_{0 y} \lambda_{2 x}+2 \lambda_{2 x y} \lambda_{0}+4 \lambda_{2 x} \lambda_{0} \lambda_{2}+2 \lambda_{3 y} \lambda_{0}+4 \lambda_{0} \lambda_{2} \lambda_{3}+\lambda_{1} \lambda_{5}\right) \\
& \quad-\lambda_{3}^{2}\left(6 \lambda_{2 x}^{2} \lambda_{0}+12 \lambda_{2 x} \lambda_{0} \lambda_{3}-6 \lambda_{0 y} \lambda_{5}+6 \lambda_{0} \lambda_{3}^{2}\right)-\lambda_{4} \lambda_{5}^{2}-2 \lambda_{5}^{3}=0 .
\end{aligned}
$$

\subsubsection{Case $\lambda_{5}=0$}

Equations (22), (25), (23), (24), (19) and (20) become

$$
\begin{aligned}
& \lambda_{0 x}=-2 \lambda_{0} \lambda_{1}, \\
& \lambda_{2 x}=-\lambda_{3}, \\
& 2 G G_{y y} \lambda_{0}-6 G_{y}^{2} \lambda_{0}+2 G_{y} G\left(3 \lambda_{0 y}+2 \lambda_{0} \lambda_{2}\right)-G^{2}\left(\lambda_{4}-\lambda_{1} \lambda_{3}\right)=0 .
\end{aligned}
$$

If $\lambda_{0} \neq 0$, then equation (30) defines

$$
G_{y y}=\left(6 G_{y}^{2} \lambda_{0}-2 G_{y} G\left(3 \lambda_{0 y}+2 \lambda_{0} \lambda_{2}\right)+G^{2}\left(\lambda_{4}-\lambda_{1} \lambda_{3}\right)\right) /\left(2 G \lambda_{0}\right) .
$$

In this case, $\left(G_{y y}\right)_{x}=\left(G_{x y}\right)_{y}$ and $\left(G_{x}\right)_{y y}=\left(G_{y y}\right)_{x}$ are satisfied. Hence, there are no other compatibility conditions. Thus, if $\lambda_{3} \neq 0, \lambda_{5}=0$ and $\lambda_{0} \neq 0$, then conditions (28) and (29) are sufficient for equation (5) to be linearizable by a generalized Sundman transformation.

If $\lambda_{0}=0$, there is no other conditions.

Remark 1. If $\lambda_{5}=0$, equations (22), (23), (24), (25) and (27) become conditions (28) and (29) respectively.

Thus, sufficient conditions for equation (5) in the case $\lambda_{3} \neq 0$ to be linearizable by generalized Sundman transformations are (22), (23), (24) and (27). 


\subsection{Case $\lambda_{3}=0$}

Notice that the particular case $\lambda_{3}=0$ and $\lambda_{4}=0$ was studied in [4]. Here the case $\lambda_{3}=0$ and $\lambda_{4} \neq 0$ is considered.

Equation (20) for $\lambda_{3}=0$ becomes

$$
2 G G_{y y} \lambda_{0}-6 G_{y}^{2} \lambda_{0}+2 G_{y} G\left(3 \lambda_{0 y}+2 \lambda_{0} \lambda_{2}\right)-G^{2} \lambda_{4}=0 .
$$

The assumption $\lambda_{0}=0$ leads to the contradiction that $\lambda_{4}=0$. Hence, one has to assume that $\lambda_{0} \neq 0$.

Equations (18), (15) and (19) become

$$
\begin{aligned}
& \lambda_{2 x x}=-\lambda_{2 x} \lambda_{1}, \\
& G_{x}=\left(G \lambda_{0 x}\right) /\left(2 \lambda_{0}\right), \\
& G_{y} \lambda_{0} \lambda_{6}-G\left(\lambda_{6 y} \lambda_{0}-\lambda_{0 y} \lambda_{6}\right)=0,
\end{aligned}
$$

where

$$
\lambda_{6}=\lambda_{0 x}+2 \lambda_{0} \lambda_{1}
$$

Substituting $G_{x}$ into equations (14) and (13), one gets

$$
\begin{aligned}
& \lambda_{6 y}=\left(\lambda_{0 y} \lambda_{6}+2 \lambda_{2 x} \lambda_{0}^{2}\right) / \lambda_{0}, \\
& \lambda_{6 x}=\left(3 \lambda_{6}\left(\lambda_{6}-2 \lambda_{0} \lambda_{1}\right)\right) /\left(2 \lambda_{0}\right) .
\end{aligned}
$$

\subsubsection{Case $\lambda_{6} \neq 0$}

From equations (35), one finds

$$
G_{y}=G\left(-\lambda_{0 y} \lambda_{6}+\lambda_{6 y} \lambda_{0}\right) /\left(\lambda_{0} \lambda_{6}\right) .
$$

Substituting $G_{y}$ into equations (14) and (32), and comparing the mixed derivatives $\left(G_{x}\right)_{y}=$ $\left(G_{y}\right)_{x}$, one obtains

$$
\lambda_{4 x}=\left(-24 \lambda_{2 x}^{2} \lambda_{0}^{3}-4 \lambda_{0} \lambda_{1} \lambda_{4} \lambda_{6}+\lambda_{4} \lambda_{6}^{2}\right) /\left(2 \lambda_{0} \lambda_{6}\right) .
$$

\subsubsection{Case $\lambda_{6}=0$}

In this case equation (35) is satisfied. One needs to check the only condition $\left(G_{y y}\right)_{x}=\left(G_{x}\right)_{y y}$, which is

$$
\lambda_{4 x}=-2 \lambda_{1} \lambda_{4}
$$

Equation (36) becomes

$$
\lambda_{2 x}=0 .
$$

Remark 2. If $\lambda_{6}=0$, equation (36) becomes a condition (40).

All obtained results can be summarized in the theorem.

Theorem 1. Sufficient conditions for equation (5) to be linearizable via a generalized Sundman transformation with $F_{x}=0$ are as follows.

(a) If $\lambda_{3} \neq 0$, then the conditions are (22), (23), (24) and (27). 
(b) If $\lambda_{3}=0, \lambda_{6} \neq 0$, then the conditions are (33), (36), (37) and (38).

(c) If $\lambda_{3}=0, \lambda_{6}=0$, then the conditions are (33), (36), (37) and (39).

Remark 3. These conditions extend the criteria obtained in [4] to the case $\alpha, \beta, \gamma \neq 0$ in (3), for restricted $\left(F_{x}=0\right)$ generalized Sundman transformations.

Remark 4. Notice that a discussion of the case $\lambda_{i}=\lambda_{i}(y)(i=0,1,2)$ and $G_{x}=0$, is also given in [7].

Remark 5. Recall S. Lie's results [3] on linearization of a second-order ordinary differential equation via a change of the independent and dependent variables (point transformations). The necessary form of a linearizable equation $y^{\prime \prime}=f\left(x, y, y^{\prime}\right)$ has to be the following form

$$
y^{\prime \prime}+a(x, y) y^{\prime 3}+b(x, y) y^{\prime 2}+c(x, y) y^{\prime}+d(x, y)=0 .
$$

Equation (41) is linearizable if and only if its coefficients satisfy the conditions

$$
\begin{aligned}
& 3 a_{x x}-2 b_{x y}+c_{y y}-3 a_{x} c+3 a_{y} d+2 b_{x} b-3 c_{x} a-c_{y} b+6 d_{y} a=0, \\
& b_{x x}-2 c_{x y}+3 d_{y y}-6 a_{x} d+b_{x} c+3 b_{y} d-2 c_{y} c-3 d_{x} a+3 d_{y} b=0 .
\end{aligned}
$$

Despite that the form (5) is a particular case of (41), sufficient conditions of linearization via point transformations (42) and the generalized Sundman transformation differ. Hence, the second class of equations is not contained in the first class due to differences in conditions on arbitrary elements of the classes. At the same time, these classes have a nonempty intersection.

\section{Examples}

Example 1. Consider the nonlinear ordinary differential equation

$$
y^{\prime \prime}+(1 / y) y^{\prime 2}+y y^{\prime}+1 / 2=0 \text {. }
$$

Since this equation does not satisfy Lie criteria for linearization [3] it is not linearizable by point transformations. Equation (43) is of the form (5) with coefficients

$$
\lambda_{2}=1 / y, \quad \lambda_{1}=y, \quad \lambda_{0}=1 / 2 .
$$

One can check that the coefficients (44) obey the conditions (22), (23), (24) and (27). Thus, equation (43) is linearizable via generalized Sundman transformation.

For finding the functions $F$ and $G$ one has to solve equations (9), (21) and (26), which become

$$
F_{x}=0, \quad F_{y y}=\left(2 F_{y}\right) / y, \quad G_{x}=0, \quad G_{y}=G / y .
$$

We take the simplest solution, $F=y^{3}$ and $G=y$, which satisfies (9), (21) and (26). One obtains the transformation

$$
u=y^{3}, \quad d t=y d x .
$$

Equations (10), (11) and (16) give

$$
\beta=1, \quad \gamma=-3 / 2, \quad \alpha=0 .
$$

Hence equation (43) is mapped by the transformation (45) into the linear equation

$$
u^{\prime \prime}+u^{\prime}+3 / 2=0 .
$$


The general solution of equation (46) is

$$
u=c_{1}+c_{2} e^{-t}-3 t / 2,
$$

where $c_{1}, c_{2}$ are arbitrary constants. Applying the generalized Sundman transformation (45) to equation (43) one obtains that the general solution of equation (43) is

$$
y(x)=\left(c_{1}+c_{2} e^{-\phi(x)}-3 \phi(x) / 2\right)^{1 / 3},
$$

where the function $t=\phi(x)$ is a solution of the equation

$$
\frac{d t}{d x}=\left(c_{1}+c_{2} e^{-t}-3 t / 2\right)^{1 / 3} .
$$

For example, if $c_{1}=c_{2}=0$, then one obtains the solution of equation (43):

$$
y=(-x)^{1 / 2} \text {. }
$$

Example 2. Consider the nonlinear ordinary differential equation

$$
y^{\prime \prime}+x y^{\prime 2}+y y^{\prime}+1 / e^{2 x y}=0 .
$$

Equation (47) is of the form (5) with the coefficients

$$
\lambda_{2}=x, \quad \lambda_{1}=y, \quad \lambda_{0}=1 / e^{2 x y} .
$$

One can check that the coefficients (48) do not satisfy the conditions of linearizability by point transformations, but they obey the conditions (28) and (29). Thus, equation (47) is linearizable via a generalized Sundman transformation.

For finding the functions $F$ and $G$ one has to solve equations (9), (21) and (31), which become

$$
\begin{aligned}
& F_{x}=0, \quad F_{y y}=\left(G_{y} F_{y}+F_{y} G x\right) / G, \\
& G_{x}=-y G, \quad G_{y y}=\left(3 G_{y}^{2}+4 G_{y} G x+2 G^{2} x^{2}\right) / G .
\end{aligned}
$$

We take the simplest solution, $F=y$ and $G=e^{-x y}$, which satisfies (9), (21) and (31). The linearizing generalized Sundman transformation is

$$
u=y, \quad d t=e^{-x y} d x .
$$

Equations (10), (11) and (16) give

$$
\beta=0, \quad \gamma=-1, \quad \alpha=0 .
$$

Hence equation (47) is mapped by the transformation (49) into the linear equation

$$
u^{\prime \prime}+1=0 \text {. }
$$

The general solution of equation (50) is

$$
u=-t^{2} / 2+c_{1} t+c_{2}
$$

where $c_{1}, c_{2}$ are arbitrary constants. Applying the generalized Sundman transformation (49) to equation (47) one obtains that the general solution of equation (47) is

$$
y(x)=-\phi(x)^{2} / 2+c_{1} \phi(x)+c_{2},
$$

where the function $t=\phi(x)$ is a solution of the equation

$$
\frac{d t}{d x}=e^{-x\left(-t^{2} / 2+c_{1} t+c_{2}\right)}
$$


Example 3. Consider the nonlinear second-order ordinary differential equation

$$
y^{\prime \prime}+\mu_{3} y^{k_{3}} y^{\prime 2}+\mu_{2} y^{k_{2}} y^{\prime}+\mu_{1} y^{k_{1}}=0,
$$

where $k_{1}, k_{2}, k_{3}, \mu_{1}, \mu_{2}$ and $\mu_{3} \neq 0$ are arbitrary constants. The Lie criteria [3] show that the nonlinear equation (51) is linearizable by a point transformation if and only if $\mu_{1}=0$ and $\mu_{2}=0$.

From equation (51), the coefficients are

$$
\begin{aligned}
& \lambda_{0}=\mu_{1} y^{k_{1}}, \quad \lambda_{1}=\mu_{2} y^{k_{2}}, \quad \lambda_{2}=\mu_{3} y^{k_{3}}, \quad \lambda_{3}=\mu_{2} k_{2} y^{k_{2}} / y, \\
& \lambda_{4}=2 \mu_{1} y^{\left(k_{1}+k_{3}\right)+1}\left(k_{1} \mu_{3}+k_{3} \mu_{1}\right)+2 \mu_{1} y^{k_{1}}\left(k_{1}^{2}-k_{1}\right)-k_{2} \mu_{2}^{2} y^{2 k_{2}+1} / y^{2}, \\
& \lambda_{5}=k_{2} \mu_{2}^{2} y^{2 k_{2}} / y .
\end{aligned}
$$

If $\mu_{2} \neq 0$ and $\mu_{1}=0$, then $\lambda_{3} \neq 0$ and $\lambda_{5} \neq 0$. One can check that the coefficients obey the conditions (22), (23), (24) and (27). Thus, equation

$$
y^{\prime \prime}+\mu_{3} y^{k_{3}} y^{\prime 2}+\mu_{2} y^{k_{2}} y^{\prime}=0
$$

is linearizable by a generalized Sundman transformation.

For finding the functions $F$ and $G$ one has to solve equations (9), (21) and (26), which become

$$
F_{x}=0, \quad F_{y y}=F_{y}\left(\mu_{3} y^{k_{3}+1}+k_{2}\right) / y, G_{x}=0, \quad G_{y}=G k_{2} / y .
$$

For example, if $k_{2}=k_{3}$, one takes the simplest solution, $F=\frac{1}{\mu_{3}} e^{\frac{\mu_{3} k_{2}+1}{k_{2}+1}}$ and $G=y^{k_{2}}$, and the generalized Sundman transformation becomes

$$
u=\frac{1}{\mu_{3}} e^{\frac{\mu_{3} y^{k_{2}+1}}{k_{2}+1}}, \quad d t=y^{k_{2}} d x .
$$

Equations (10), (11) and (16) give

$$
\beta=\mu_{2}, \quad \gamma=0, \quad \alpha=0 .
$$

Hence equation (52) is mapped by the transformation (53) into the linear equation

$$
u^{\prime \prime}+\mu_{2} u^{\prime}=0 .
$$

If $\mu_{3}=0$, then equation (51) is

$$
y^{\prime \prime}+\mu_{2} y^{k_{2}} y^{\prime}+\mu_{1} y^{k_{1}}=0,
$$

where $\mu_{2} \neq 0$. The Lie criteria [3] show that the nonlinear equation (54) is linearizable by a point transformation if and only if $k_{1}=3, k_{2}=1$ and $\mu_{1}=\left(\mu_{2} / 3\right)^{2}$. In the particular case, $k_{1}=3, k_{2}=1, \mu_{1}=1$ and $\mu_{2}=3$, one has the equation

$$
y^{\prime \prime}+3 y y^{\prime}+y^{3}=0 \text {. }
$$

Equation (55) arises in many areas. Some of these are the analysis of the fusion of pellets, the theory of univalent functions, the stability of gaseous spheres, operator Yang-Baxter equations, motion of a free particle in a space of constant curvature, the stationary reduction of the second member of the Burgers hierarchy [9].

Remark 6. Equation (55) is linearizable by a point transformation and by a generalized Sundman transformation into the equation $u^{\prime \prime}=0$ and $u^{\prime \prime}+3 u^{\prime}+2 u=0$, respectively. 
Without loss of the generality ${ }^{3}$, one can assume that $\mu_{2}=1$. Hence, equation (54) becomes

$$
y^{\prime \prime}+y^{k_{2}} y^{\prime}+\mu_{1} y^{k_{1}}=0 .
$$

For this equation the coefficients are

$$
\begin{aligned}
& \lambda_{0}=\mu_{1} y^{k_{1}}, \quad \lambda_{1}=y^{k_{2}}, \quad \lambda_{2}=0, \quad \lambda_{3}=k_{2} y^{k_{2}-1}, \\
& \lambda_{4}=\mu_{1} k_{1}\left(k_{1}-1\right) y^{k_{1}-2}-k_{2} y^{2 k_{2}-1}, \quad \lambda_{5}=k_{2} y^{2 k_{2}+1} .
\end{aligned}
$$

If $k_{2}=0$, then $\lambda_{5}=0$ and equation (56) is linearizable by a generalized Sundman transformation.

If $k_{2} \neq 0$, then $\lambda_{5} \neq 0$ and conditions (22), (23), (24), (27) are reduced to

$$
\mu_{1}\left(2 k_{2}+1-k_{1}\right)\left(k_{2}-k_{1}\right)=0 \text {. }
$$

If conditions (57) are satisfied, then equation (56) is linearizable by a generalized Sundman transformation. Notice that in the case $\mu_{1}\left(k_{2}-k_{1}\right)=0$, equation (56) is trivially integrated by using the substitution $y^{\prime}=H(y)$. A nontrivial case is $k_{1}=2 k_{2}+1$. In this case the functions $F$ and $G$ are solutions of the compatible overdetermined system of equations

$$
F_{x}=0, \quad F_{y y}=k_{2} F_{y} / y, \quad G_{x}=0, \quad G_{y}=k_{2} G / y
$$

The general solution of equations (58) depends on the value of the constant $k_{2}$. For example, if $k_{2} \neq-1$, then a particular solution of system (58) is

$$
F=y^{k_{2}+1}, \quad G=y^{k_{2}} .
$$

Thus, the generalized Sundman transformation reduces equation (56) into the linear equation

$$
u^{\prime \prime}+u^{\prime}+\left(\mu_{1}\left(k_{2}+1\right)\right) u=0 .
$$

Remark 7. Since equations (43), (51) and (54) are autonomous, their order can be reduced by the substitution $y^{\prime}=f(y)$. It is worth to note that for equations (51) and (54) the difficulties in using the generalized Sundman transformation are similar to solving the original equation by this reduction.

\section{Conclusion}

Application of the generalized Sundman transformation for the linearization problem was analyzed in the paper. Since the method is well-known, the efficiency of the method is not discussed in the paper. The paper just warns that a researcher has to be careful when using the wellknown method for the linearization problem. In particular, our examples show that, in contrast to point transformations (S. Lie results), for a linearization problem via the generalized Sundman transformation one needs to use the general form of a linear second-order ordinary differential equation instead of the Laguerre form.

\section{Acknowledgements}

This research was supported by the Royal Golden Jubilee Ph.D. Program of Thailand (TRF).

\footnotetext{
${ }^{3}$ For example, scaling of the independent variable: $\bar{x}=\mu_{2} x$.
} 


\section{References}

[1] Meleshko S.V., Methods for constructing exact solutions of partial differential equations, Mathematical and Analytical Techniques with Applications to Engineering, Springer, New York, 2005.

[2] Ibragimov N.H., Elementary Lie group analysis and ordinary differential equations, Wiley Series in Mathematical Methods in Practice, Vol. 4, John Wiley \& Sons, Ltd., Chichester, 1999.

[3] Lie S., Classifikation und Integration von gewöhnlichen Differentialgleichungen zwischen $x, y$ die eine Gruppe von Transformationen gestatten, Math. Ann. 32 (1888), 213-281.

[4] Duarte L.G.S., Moreira I.C., Santos F.C., Linearization under non-point transformations, J. Phys. A: Math. Gen. 27 (1994), L739-L743.

[5] Euler N., Wolf T., Leach P.G.L., Euler M., Linearisable third-order ordinary differential equations and generalised Sundman transformations: the case $X^{\prime \prime \prime}=0$, Acta Appl. Math. 76 (2003), 89-115, nlin.SI/0203028.

[6] Nakpim W., Meleshko S.V., Linearization of third-order ordinary differential equations by generalized Sundman transformations: the case $X^{\prime \prime \prime}+\alpha X=0$, Commun. Nonlinear Sci. Numer. Simul. 15 (2010), 1717-1723.

[7] Berkovich L.M., The integration of ordinary differential equations: factorization and transformations, Math. Comput. Simulation 57 (2001), 175-195.

[8] Berkovich L.M., Factorization and transformations of differential equations. Methods and applications, R\&C Dynamics, Moscow, 2002 (in Russian).

[9] Karasu A., Leach P.G.L., Nonlocal symmetries and integrable ordinary differential equations: $\ddot{x}+3 x \dot{x}+x^{3}=0$ and its generalizations, J. Math. Phys. 50 (2009), 073509, 17 pages. 